\title{
Brain Dynamics in Predicting Driving Fatigue Using a Recurrent Self-Evolving Fuzzy Neural Network
}

\author{
Yu-Ting Liu, Yang-Yin Lin, Member, IEEE, Shang-Lin Wu, Member, IEEE, Chun-Hsiang Chuang and \\ Chin-Teng Lin, Fellow, IEEE
}

\begin{abstract}
This study proposes a generalized prediction system called a recurrent self-evolving fuzzy neural network (RSEFNN) that employs an on-line gradient descent (GD) learning rule to address the electroencephalography (EEG) regression problem in brain dynamics for driving fatigue. The cognitive states of drivers significantly affect driving safety; in particular, fatigue driving, or drowsy driving, endangers both the individual and the public. For this reason, the development of brain-computer interfaces (BCIs) that can identify drowsy driving states is a crucial and urgent topic of study. Many EEG-based BCIs have been developed as artificial auxiliary systems for use in various practical applications because of the benefits of measuring EEG signals. In the literature, the efficacy of EEG-based BCIs in recognition tasks has been limited by low resolutions. The system proposed in this paper represents the first attempt to utilize the recurrent fuzzy neural network (RFNN) architecture to increase adaptability in realistic EEG applications to overcome this bottleneck. This study further analyzes brain dynamics in a simulated car driving task in a virtual-reality (VR) environment. The proposed RSEFNN model is evaluated using the generalized cross-subject approach, and the results indicate that the RSEFNN is superior to competing models regardless of the use of recurrent or non-recurrent structures.
\end{abstract}

This work was supported in part by the Aiming for the Top University Plan of National Chiao Tung University, sponsored by the Ministry of Education, Taiwan, under Grant Number 104W963; in part by the UST-UCSD International Center of Excellence in Advanced Bio-engineering, sponsored by the Ministry of Science and Technology I-RiCE Program under Grant Number MOST 103-2911-I-009-101; in part by MOST 104-2627-E-009-001; in part by the Cognition and Neuroergonomics Collaborative Technology Alliance Annual Program Plan, sponsored by the Army Research Laboratory under Cooperative Agreement Number W911NF-10-2-0022; and in part by the Tung Thih Electronic Fellowship.

Yu-Ting Liu and Shang-Lin Wu are with the Institute of Electrical Control Engineering, National Chiao Tung University, Hsinchu 30010, Taiwan (e-mail: tingting76319@gmail.com; slwu19870511@gmail.com).

Yang-Yin Lin is with the Electronic Systems Research Division, National Chung-Shan Institute of Science and Technology (NCSIST), Taoyuan 32546, Taiwan (e-mail: oliver.yylin@gmail.com).

Chun-Hsiang Chuang is with the Brain Research Center, National Chiao Tung University, Hsinchu 30010, Taiwan; the Faculty of Engineering and Information Technology, University of Technology Sydney, Sydney 2007, Australia (e-mail: chchuang@ieee.org).

*Chin-Teng Lin is with the Brain Research Center and the Lifetime Chair Professor of Electrical Engineering and Computer Science, National Chiao Tung University, Hsinchu 30010, Taiwan; the Faculty of Engineering and Information Technology, University of Technology Sydney, Sydney 2007, Australia (e-mail: ctlin@mail.nctu.edu.tw).
Index Terms-Electroencephalography (EEG), Driving Fatigue, Recurrent Fuzzy Neural Network (RFNN), Brain-Computer Interface (BCI)

\section{INTRODUCTION}

TNIVIDUALS typically possess varying degrees of driving Lexperience, and driving safety has proven to be one of the most important issues to address in the realm of public safety. Driving safety is influenced by two main factors: the external environment and the mental states of the drivers [1]. External factors are usually unpredictable and difficult to address, and doing so often incurs substantial costs. However, concerning the mental states of drivers, although investigations of the human mind remain difficult, the plentiful and substantial results obtained in the field of cognitive neuroscience have recently provided an opportunity to resolve this problem [2].

Various physiological/psychological conditions, e.g., fatigue [3], distraction [4] and motion sickness [5], can affect driving safety. Fatigue driving, or drowsy driving, exposes personal and public property to dangerous situations. Individuals may find themselves in a drowsy or fatigue state as a result of sleep deficits, long-term driving, midnight driving, monotonous driving, taking sleeping drugs, or sleep disorders [3], [6]. Long-term driving is a common cause of accidents and has been experienced by numerous drivers. Therefore, the development of artificial auxiliary systems for detecting drowsy driving states is of the utmost importance. In contrast to various other types of systems [7], [8], brain-computer interfaces (BCIs) are effective because they can directly evaluate the cognitive states of human beings [8], [9]. Many measurement methodologies have been proposed for the estimation of brain dynamics (e.g., computer tomography (CT), positron emission tomography (PET), electroencephalography (EEG), magnetoencephalography (MEG), and magnetic resonance imaging (MRI)). As one of the most important of these methodologies, EEG has attracted substantial amounts of attention over many years. A significant advantage of EEG over other extraction methodologies is that it provides convenient real-time measurements. Therefore, EEG signals are commonly used in real-world applications [10]-[12].

A considerable body of literature in cognitive science indicates that we have grasped the connections between brain 
activation and a variety of tasks through the use of EEG topography. In the literature related to driving tasks [13]-[16], EEG spectra have been widely exploited and are commonly used to identify different cognitive states. The magnitude of EEG power in the alpha band in the occipital cortex has been found to be one of the significant features that accompanies the onset of drowsiness [15]. In [13], the authors illustrated that the brain dynamics in the central and occipital lobes could be utilized to identify the difference between attention and mental fatigue based on the response time (RT) in a simulated experiment. In [14], the authors demonstrated that the lower frequency band of tonic EEG power in the occipital cortex increased during a simulated visual tracking task. Based on these fundamental findings in cognitive neuroscience, we chose to further explore and analyze the occipital region in this study.

Many BCI systems have been developed for real-life applications over the past decade because of the results of investigations in the field of cognition neuro-engineering and substantial improvements in EEG measurement technologies [8]-[12], [17]. In previous studies [3], [11], several researchers have proposed a variety of experimental assumptions and models to investigate cognitive states during vehicle driving tasks using various simulation environments. Artificial neural networks (ANNs) have been found to be potential means of addressing these problems by virtue of their unique characteristics, including massive parallelism, self-organization, adaptive learning capability and robustness [18]-[20]. However, a major difficulty related to ANNs is that the number of hidden neurons directly and strongly affects their performance; therefore, traditional neural network approaches, such as multilayer perceptron for time-varying systems or signals, are not very appropriate because of their static structure. To address this problem, fuzzy neural networks (FNNs) have been considered as a flexible and rational alternative approach because they combine bio-inspired learning with the mechanism of human thinking [11], [21], [22].

In our previous study [11], we utilized a virtual reality (VR)-based scenario to simulate different driving environments for participants and to predict the levels of fatigue driving experienced by the participants via an FNN with pre-event EEG signals. We developed a real-time system for fatigue prediction using an on-line learning algorithm with a single trial and treated each trial as an independent event. The results presented in [11], based on the FNN model, indicated superb performance compared with other fundamental types of ANNs, e.g., multilayer perceptron neural networks (MLPNNs) and radial basis function neural networks (RBFNNs).

To further address arduous problems of pattern regression/classification, recurrent models are often employed because they can exhibit various types of memory. Recurrent neural networks (RNNs) are biologically more plausible and computationally more powerful than feedforward networks, and their physical characteristics are very appropriate for modeling nonlinear dynamic systems, which use feedback connections to memorize the temporal characteristics of an incoming dataset. Recurrent connections allow the hidden neurons of the network to refer to their previous states, allowing (a)

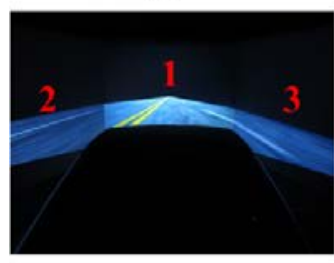

(c)

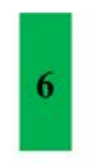

(d)

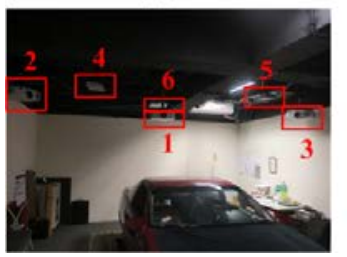

Fig. 1. Experimental setup for a virtual-reality-based experimental environment: (a) the front view of the driver in a VR scene, (b) a real car controlled by a motion platform, (c) a schematic diagram of a $360^{\circ}$ VR scene projected by six projectors, (d) the projector platform, and (e) a participant in a four-lane divided highway VR scene.

subsequent behavior to be shaped by previous responses. These recurrent connections endow the network with memory. Combined with nonlinear activation functions, RNNs are capable of processing complex spatiotemporal patterns. Therefore, RNNs were considered for this particular study over other EEG data analysis techniques for several reasons [23], [24]. An important benefit of RNNs is their ability to adapt to changes in time sequences by modifying their connection weights. This property is especially useful for analyzing signals that may exhibit changes in behavior at unpredicted times. In addition, RNNs are a specific type of architecture consisting of neurons that utilize feedback connections, and these types of neural networks can effectively capture nonlinear dynamics. Unlike static neural networks, RNNs, which possess both current and past information about temporal sequences, have proven to be a satisfactory alternative neural network approach to EEG-related studies [23]-[26].

In [10], [11], [27], the authors ignored the correlation and continuity of cognitive states in driving tasks. However, the experienced level of fatigue should form a continuous sequence in the temporal domain, and thus, researchers should integrate long-term information when developing real-time BCI systems for fatigue sensing. Furthermore, as seen by considering existing BCI systems, this paper is the first attempt to utilize such a concept while adopting the recurrent fuzzy neural network (RFNN) architecture in a realistic EEG application. A real-time RFNN was temporally trained using pre-stimulus 


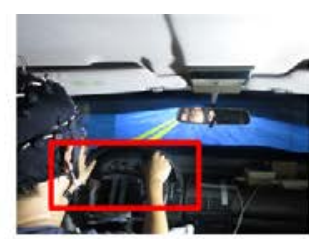

Cruising

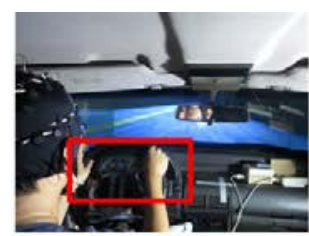

Deviation onset

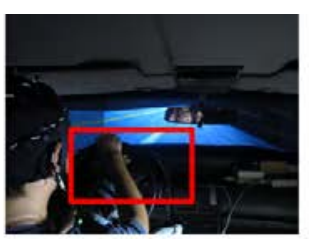

Response onset

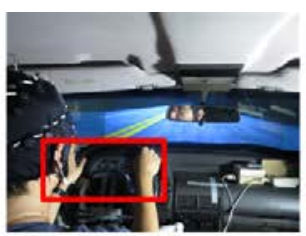

Response offect

\& Cruising
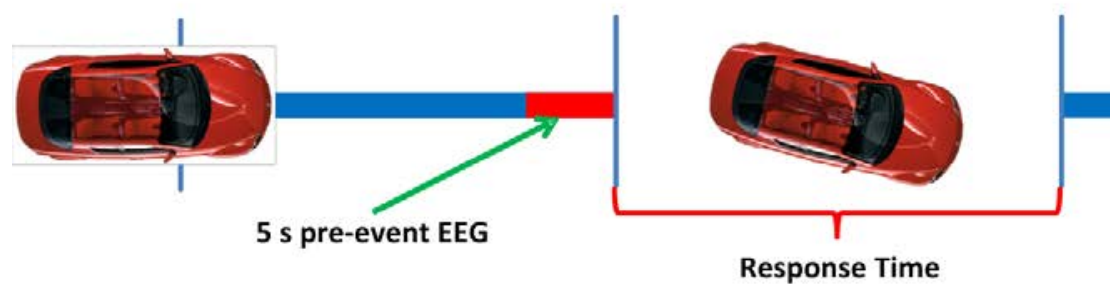

Fig. 2. Virtual-reality-based experimental highway environment with a lane-departure paradigm.

EEG activity. An RFNN combines the merits of both RNNs and FNNs, which are capable of representing and strongly encoding current/past states, and incorporates high-level human-like thinking using neurons. The BCI system proposed in this paper employs a recurrent self-evolving fuzzy neural network (RSEFNN) with local feedback [28] to classify cognitive states. In [28], such an RSEFNN was validated for use in the identification of problems in dynamical systems. In this study, a local feedback approach was implemented in the RSEFNN to enable the network to effectively memorize both past and current EEG states to further enhance the performance of the system for the analysis of nonlinear time-varying signals. Such states have never previously been applied for fatigue detection in RFNNs or, to the best of our knowledge, EEG analyses. In the proposed system, the free parameters of the RSEFNN are learned via an on-line gradient descent algorithm to enable real-time modeling for human fatigue monitoring. The locally recurrent structure used in the proposed RSEFNN is simpler than those of other existing RFNNs [29], [30], and this simple structure makes the RSEFNN better suited for use in real-time BCI applications.

We developed a driving environment simulation with six-axis motion platforms to analyze the driving tasks performed by the study participants [11], [14]. While acting as drivers, the participants were required to wear an EEG cap, which was used to observe their cognitive states during the simulated long-term driving task. The prediction systems proposed in previous studies [10], [11], [27] have focused on a specific driving task (fatigue/motion sickness) for each subject, i.e., the structures trained using the proposed algorithms are suitable only for the particular drivers who participated in those experiments. Consequently, these prediction systems might not be generalizable to other drivers. Hence, to ensure the feasibility and utility of the prediction system, this study proposes the use of an EEG-based RSEFNN to estimate driver response times to accidental events based on neurophysiological activity. We investigated a general cross-subject model of fatigue prediction in our simulated driving environment. Moreover, the proposed system was compared with various benchmark systems, including support vector regression (SVR) [31], a self-organizing neural fuzzy inference network (SONFIN) [32], a fuzzy wavelet neural network (FWNN) [33], a TSK-type recurrent fuzzy network (TRFN) [30] and a recurrent wavelet-based Elman neural network (RWENN) [34]. The experimental results indicated that the proposed recurrent system with local feedback outperformed the other systems in terms of prediction error in the generalized cross-subject model. In addition, our model required fewer feature dimensions, which reduced the computational time required during the training process. This advantage of the proposed BCI is promising for its application to real-time vehicle driving prediction in the real world.

The remainder of this paper is organized as follows. Section II presents the experimental setup, including the VR environment, the experimental paradigm, the participants and the brain dynamics measurements. Section III introduces the EEG processing procedure. Section IV presents a description of the structure of and parameter-learning methods for the RSEFNN. Section V introduces the state-of-the-art models that are used in the drowsiness prediction system. Section VI discusses the experimental results that were obtained using the proposed system. Finally, Section VII offers concluding remarks.

\section{EXPERIMENTAL SETUP}

\section{A. Virtual Reality-based Experimental Highway Environment}

The experimental environment in this study was constructed through a virtual reality (VR) scenario, as depicted in Figs. 1(a)-(d). This simulator was designed for use in our previous studies [11], [14], [16] to observe drivers' cognitive states during a variety of vehicle driving tasks. The setup of the VR environment consisted of three-dimensional images of the vehicle's surroundings projected by six projectors and a real car controlled by a six-axis motion platform, as indicated in Figs. 1(b) and (d). All scenes in this study simulated the participants driving on a four-lane divided highway at a constant speed of $100 \mathrm{~km} / \mathrm{hr}$ at night. During the experimental period, all participants were instructed to enter the car and then steer the vehicle according to the provided instructions, as displayed in Fig. 1(e). The experimental setup allowed the researchers to investigate the fatigue states of the participants during 


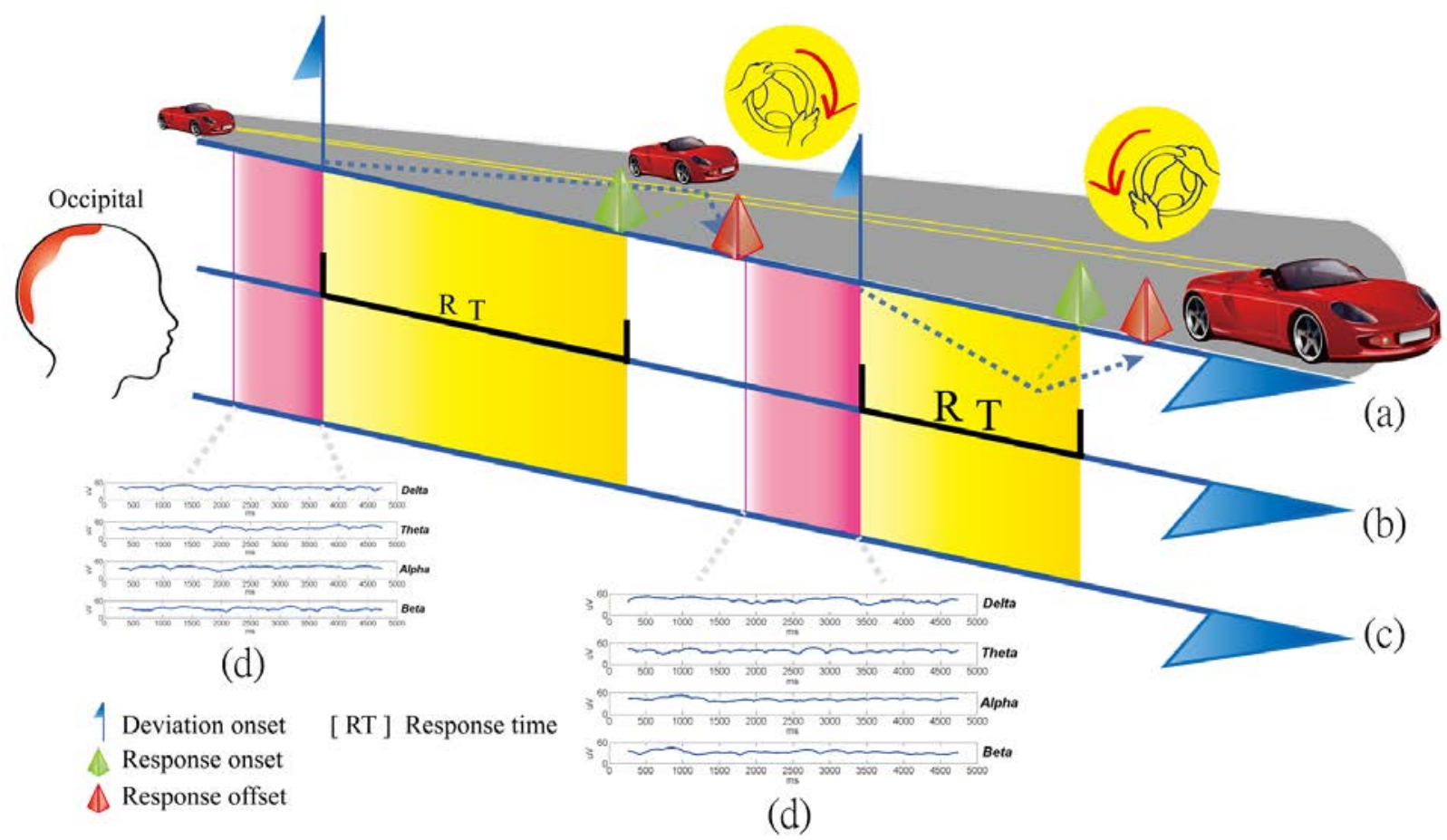

Fig. 3. Driving fatigue paradigm: (a) event-related lane-departure driving paradigm, (b) behavioral performance (yellow region) during the paradigm, (c) $5 \mathrm{~s}$ of EEG data collected from the occipital lobe before deviation onset (red region), and (d) EEG spectra of each frequency band (Delta, Theta, Alpha and Beta).

long-term driving on a highway in a realistic simulation.

\section{B. Driving Fatigue Paradigm}

To evaluate the brain dynamics occurring during the driving task, we adopted an event-related lane-departure driving paradigm based on the VR platform described above, as illustrated in Fig. 2 and Fig. 3. In the VR driving paradigm, the participants were instructed to drive within the simulated environment and to maintain the positioning of the virtual vehicle in the center of the cruising lane. Lane perturbation events were then randomly introduced to cause the virtual vehicle to drift from the center of the cruising lane. The participants, who had been trained prior to the experiment, were required to steer the vehicle back to the center of the cruising lane as soon as possible after becoming aware of the deviation. If the participants did not respond to the lane perturbation event, as a consequence of falling asleep, for example, the vehicle would hit the left and right curb of the roadside within 2.5 and $1.5 \mathrm{~s}$, respectively. The vehicle would then continue to move along the curb until it returned to the original lane. The inter-trial intervals of random lane perturbation events were set to $5 \sim 10 \mathrm{~s}$. To maintain consistency in the standard cognitive psychological state assumed for all participants, the experiment was conducted in the early afternoon (13:00-14:00) after lunch, at the peak of the expected level of sleepiness in the circadian rhythm, and lasted approximately 90 minutes. The cognitive states and fatigue levels of the participants were monitored throughout the experiment by means of a surveillance camera and the vehicle trajectory.

\section{Participants}

Twenty right-handed, healthy young adults participated in the behavioral experiment (mean age \pm standard deviation: 23.6 \pm 2.9 years old). All participants were recruited through an online advertisement. No subject had a history of neurological, psychiatric or addictive disorders, according to self-reports, and no subject had taken anti-psychotic or other relevant psychoactive drugs in the two preceding weeks. To ensure proper evaluation of their driving performance, the participants attended a pre-test session to verify that none of them was afflicted with simulator sickness. The Institutional Review Board of the Veterans General Hospital, Taipei, Taiwan, approved the study. All participants were asked to read and sign an informed consent form before participating in the EEG experiments.

\section{Brain Dynamics Measurements}

In this study, EEG was utilized to measure each participant's brain dynamics during the driving fatigue task. The EEG signals were captured from 33 sintered Ag/AgCl EEG/EOG active electrode sites with a unipolar reference and 2 ECG channels with a bipolar connection, which were all referred to linked mastoids. All EEG/EOG electrodes referred to the right ear lobe were placed in accordance with a modified International 10-20 system of electrode placement. To reduce disturbances caused by noise in the measured EEG signals, the contact impedance between the EEG electrodes and the cortex was calibrated to be less than $5 \mathrm{k} \Omega$ before recording. During the recording of the EEG/EOG/ECG data using a NeuroScan NuAmps Express system (Compumedics Ltd., VIC, Australia), the lane deviation (LD) and response time (RT) of each subject 


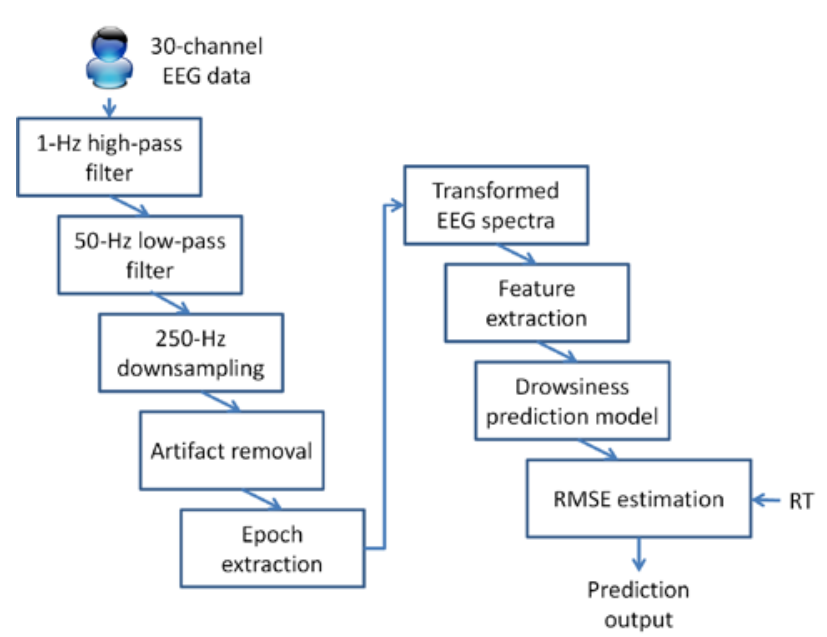

Fig. 4. System flowchart of the proposed prediction system for drowsiness levels.

were also recorded simultaneously. The RT represented the time period between the onset of the deviation and the onset of the response and was used as an objective measure of the drowsiness (DS) level during each lane-departure event. The EEG data were recorded with 16-bit quantization at a sampling rate of $500 \mathrm{~Hz}$, in accordance with the hardware specifications, and downsampling to $250 \mathrm{~Hz}$ was then applied to reduce the computational complexity during the subsequent computational phase.

To ensure that each subject experienced consistent DS levels throughout the entire experimental period, we normalized the DS levels of each subject to the range $[0,1]$. These normalized DS levels are presented as temporal sequences and are regarded as the desired output of the proposed system in this study.

\section{EEG PROCESSING AND CATEGORIZATION}

\section{A. EEG Preprocessing and Segmentation}

The general scheme of the EEG analysis is illustrated in Fig. 4. The acquired EEG data were processed and analyzed using EEGLAB (http://www.sccn.ucsd.edu/eeglab/, an open-source EEG toolbox for MATLAB) [35] during the preprocessing stage; the raw EEG signals were subjected to a 1-Hz high-pass and a $50-\mathrm{Hz}$ low-pass infinite impulse response (IIR) filter and then downsampled to $250 \mathrm{~Hz}$ from the sample recording rate of $500 \mathrm{~Hz}$ used during the hardware phase. The artifacts, such as one or more eye blinks or electromyography activity, were then removed from the filtered EEG data. After artifact removal, the data were sliced into segmented epochs based on the corresponding event tags associated with the recorded data.

The recorded temporal EEG sequences were then segmented into a series of epochs of dynamic sequences lasting from $5 \mathrm{~s}$ before the occurrence of each deviation to the end of the deviation event. Each extracted event was composed of three different cognitive courses during the lane perturbation event.

(1) The baseline period (the period before the onset of the deviation) is represented by the red region in Fig. 3. During this experimental period, the participants were asked to concentrate on steering the vehicle on the highway in as straight a line as possible. The EEG data recorded during this baseline period were the so-called pre-event EEG data.

(2) The lane-departure period (between the onset of the deviation and the onset of the response) is represented by the yellow region in Fig. 3. The deviation formally occurred during this period, and the participants experienced lane perturbation events that caused the virtual vehicle to drift from the center of the cruising lane.

(3) The steering period (between the onset and end of the response) is represented by the blank region in Fig. 3. After the participants became aware of the vehicle deviation, they were required to steer the vehicle back to the center of the cruising lane as soon as possible. As indicated in Fig. 3(b), this temporal period was called the RT for each lane-departure event and was treated as the indicator of the DS level in the proposed fatigue prediction system.

When the participants remained alert during the baseline period, their RTs to the random drifts were short, thus resulting in small deviations from the center of the lane. By contrast, if the participants were in a fatigue state, the RTs and lane deviations were large. In this study, the EEG data recorded $5 \mathrm{~s}$ before deviation onset served as estimation of the driver's physiological state, and the RT, which was calculated as the time between the deviation onset and the response onset, was used to define the participant's state of awareness. Using the proposed system, we utilized the features of the EEG data before each lane-departure event to predict the DS level of the participants.

\section{B. Power Spectrum Analysis and Feature Extraction}

To investigate the brain dynamics of the participants following each lane-departure event and their subsequent motor responses, each epoch in the channels related to the occipital lobe $\left(\mathrm{O}_{1}, \mathrm{O}_{2}\right.$ and $\left.\mathrm{O}_{\mathrm{z}}\right)$ was separately transformed into the time-frequency representation using the event-related spectral perturbation routine [35]. Physiological features were then extracted by transforming the time-series EEG signals into the frequency domain using a fast Fourier transform (FFT) to reveal the spectral dynamics of the brain activity. For each channel of interest associated with the cerebral cortex, the mean powers in the delta $(1-3 \mathrm{~Hz})$, theta $(4-7 \mathrm{~Hz})$, alpha $(8-12 \mathrm{~Hz})$, and beta $(13-20 \mathrm{~Hz})$ bands were collected for power spectrum analysis and feature extraction.

\section{RSEFNN FOR DRIVING FATIGUE ESTIMATION AND Prediction using PRE-EVENT EEG Signals}

Brain dynamics are a series of activations caused by a variety of internal and external stimuli. In traditional brain monitoring systems, researchers have focused on developing adaptive structures for estimating different cognitive states [10]-[12] such as motion, attention and fatigue. However, EEG identification problems typically ignore the fact that an individual's cognitive state is a product of a continuous process based on accumulated memories of previous experiences [10], [11], [18]-[22], [27]. Instead, the designers of a practical brain monitoring system should regard it as a dynamic system that considers current and past information from both the internal 


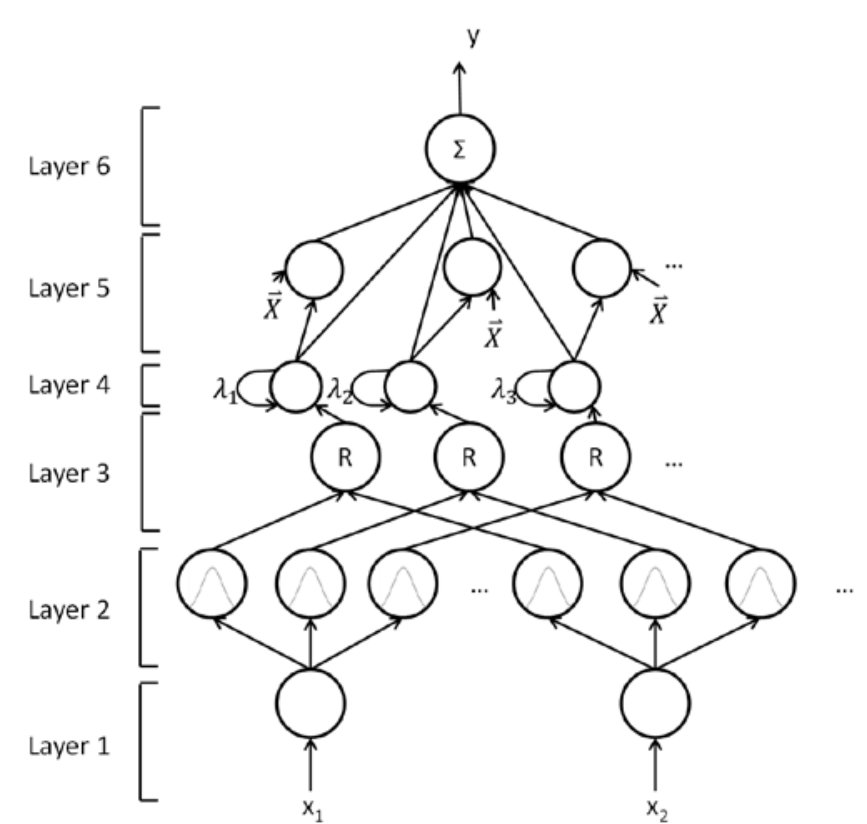

Fig. 5. Structure of the recurrent self-evolving fuzzy neural network (RSEFNN).

and external environments [23], [24], [26]. In other words, the output of a dynamic system involves past outputs, past inputs, current inputs and current outputs. To address problems related to dynamic systems, recurrent structures, which are usually constructed as closed-loop systems, are employed because of their memory capabilities. This paper represents the first attempt to utilize this concept when implementing an RFNN architecture for a realistic EEG application. An RFNN combines the merits of both RNNs and FNNs, which are capable of representing and strongly encoding current/past states, and exhibits high-level human-like thinking using neurons. RFNNs have previously been used to address time-varying system problems in [28], [30].

A recurrent self-evolving fuzzy neural network (RSEFNN) [28] with local feedback is utilized in this paper. The recurrent structure of the RSEFNN originates from locally feeding the firing strength of a fuzzy rule back to itself, thereby avoiding the use of additional external registers for memorizing past states. In contrast to other RNNs [25], [36], the computational complexity of the RSEFNN is low because of its simplified recurrent structure. The locally recurrent structure in the RSEFNN is simple, but its performance is admirable in comparison with those of other fully recurrent FNNs [28]-[30]. The RSEFNN consists of two simultaneous learning phases: structure learning and parameter learning. Here, simultaneous learning means that for each piece of incoming data, both the structure- and parameter-learning algorithms are performed on-line. Because of this simultaneous learning property, the RSEFNN can be used in normal operation at any time because learning proceeds without the need to assign any fuzzy rules in advance.

For an incoming datum, structure learning is performed first, and parameter learning is applied in a subsequent phase. Specifically, parameter learning is performed in a timely manner in the newly evolved network structure. During structure learning, a self-evolving fuzzy set generation algorithm is employed to adaptively construct fuzzy rules upon the receipt of training data. All of the fuzzy sets and rules in the RSEFNN are generated adaptively, which not only automates the rule generation process but also enables the creation of superior initial rules for subsequent parameter learning. During parameter learning, all free parameters in the antecedent and consequent components are learned through an on-line gradient descent algorithm using metrics derived from pattern sequences. The following sections introduce the details of the RSEFNN structure and the implemented learning procedures.

\section{A. Structure of the RSEFNN}

The RSEFNN is composed of six layers, where each layer contains nodes that serve as neurons via any arbitrary type of nonlinear operator. The structure of the RSEFNN model is illustrated in Fig. 5. The mathematical functions of each layer in the RSEFNN are detailed below. In the following description, $u^{(l)}$ denotes the output of a node in the $l^{\text {th }}$ layer.

Layer 1(Input layer) - The inputs are denoted by $\vec{X}=$ $\left(x_{1} \ldots x_{n}\right)$. No computations are performed in layer 1 . Each node in this layer corresponds to one input variable and simply transmits the input values directly to the next layer.

$$
u_{i}^{(1)}=x_{i}
$$

Layer 2 (Fuzzification layer) - Layer 2 is also called the membership function layer. Each node in this layer uses a Gaussian membership function that corresponds to the linguistic label of an input variable in layer 1 . The calculated membership value in layer 2 is

$$
u_{i j}^{(2)}=\exp \left(-\frac{\left[u_{i}^{(1)}-m_{i j}\right]^{2}}{\sigma_{i j}^{2}}\right)
$$

where $m_{i j}$ and $\sigma_{i j}^{2}$ are the mean and variance of the Gaussian membership function, respectively, of the $j_{t h}$ term of the $i_{t h}$ input variable $x_{i}$.

Layer 3 (Spatial firing layer) - Each node in layer 3 corresponds to one fuzzy rule and functions as a spatial rule node. The nodes in layer 3 receive single-dimensional membership degrees based on the corresponding rules from the nodes of a set in layer 2 .

Here, the fuzzy AND operator is used to perform the preconditioning of the fuzzy rules using an algebraic product operation to obtain a spatial firing strength $F^{j}$. The spatial firing strength of each rule node can thus be denoted by

$$
u_{j}^{(3)}=F^{j}=\prod_{j}\left(u_{i j}^{(2)}\right)
$$

where $\prod_{j} u_{i j}^{(2)}$ for a rule node represents the spatial firing strength of its corresponding rule. 
Layer 4 (Temporal firing layer) - Each node in layer 4 is a recurrent fuzzy rule node that forms an internal feedback loop. The time step is denoted by $t$. The output of such a recurrent node is a temporal firing strength $\phi_{q}^{j}(t)$ that incorporates both the spatial firing strength and the temporal firing strength $\phi_{q}^{j}(t-1)$. The temporal firing strength $\phi_{q}^{j}(t)$ is a linear combination of the spatial firing strength $F^{j}(t)$ and the previous temporal firing strength $\phi_{q}^{j}(t-1)$. The equation for this combination is

$$
u_{j}^{(4)}=\phi_{q}^{j}(t)=\lambda_{q}^{j} F^{j}(t)+\left(1-\lambda_{q}^{j}\right) \phi_{q}^{j}(t-1)
$$

where $0 \leq \lambda_{q}^{j} \leq 1$ is a recurrent parameter that determines the ratio between the contributions of the current and past states. The recurrent parameters are initialized with random values that are uniformly generated on the interval $[0,1]$ and then updated by a learning algorithm.

Layer 5 (Consequent layer) - The nodes in layer 5 are called consequent nodes. The weight of a link from a node in layer 4 to one in layer 5 is $a_{i q}^{j}$. Each recurrent rule node in layer 4 has a corresponding consequent node in layer 5 . The consequent node function produces a linear combination of the input variables. The output of layer 5 is computed as follows:

$$
u_{j}^{(5)}=f_{q}^{j}(t)=\sum_{i=1}^{n} a_{i q}^{j} x_{i}(t)
$$

Layer 6 (Output layer) - The output node in layer 6, which performs fuzzy defuzzification, integrates all of the actions recommended in layer 5 and the recurrent nodes in layer 4 . The weighted average defuzzification method is used in this layer:

$$
y=u_{j}^{(6)}=\frac{\sum_{j=1}^{R} \phi_{q}^{j}(t)\left(f_{q}^{j}(t)+a_{0 q}^{j}\right)}{\sum_{j=1}^{R} \phi_{q}^{j}(t)}=\frac{\sum_{j=1}^{R} \phi_{q}^{j}(t)\left(\sum_{i=0}^{n} a_{i q}^{j} x_{i}\right)}{\sum_{j=1}^{R} \phi_{q}^{j}(t)}
$$

where $\mathrm{R}$ is the total number of fuzzy rules and $\mathrm{y}$ is the output of the RSEFNN model.

\section{B. Structure Learning}

The purpose of structure learning is to determine when to generate a new fuzzy rule. Fuzzy rules are generated from the training data to ensure that at least one rule is activated with a firing strength greater than a pre-defined threshold $F_{t h} \in(0,1)$ for each input $\vec{X}$. Geometrically, this ensures that each input datum is properly covered by a rule in the input space.

For each incoming input pattern $\vec{X}$, the spatial firing strength $F^{j}(t)$ in Eq. (4) is used as the criterion for determining whether a new fuzzy rule should be generated. Initially, there are no rules in the intrinsic network. For the first incoming datum
$\vec{X}(0)$, a new fuzzy rule is generated with an initial center and width of a Gaussian membership function as follows:

$$
\begin{aligned}
& m_{i}^{1}=x_{i}(0) \\
& \sigma_{i}^{1}=\sigma_{\text {initial }}
\end{aligned}
$$

where $\sigma_{\text {initial }}$ is a pre-defined value that determines the width of each fuzzy set.

For a subsequent incoming datum $\vec{X}(t)$, we calculate the maximum spatial firing strength $F^{j}(t)$ as the indicator of whether a new fuzzy rule should be generated:

$$
I=\arg \max _{1 \leq j \leq R(\mathrm{t})} F^{j}(t)
$$

where $R(t)$ is the number of rules in the current network at time $t$. If $F^{I}(t)<F_{\text {thresh }}$, where $F_{\text {thresh }} \in(0,1)$ is a user-defined threshold, then a new fuzzy rule is generated.

When a new fuzzy rule is generated, the center and width of the new fuzzy set are set as follows:

$$
\begin{aligned}
& m_{i}^{R(t+1)}=x_{i}(t) \\
& \sigma_{i}^{R(t+1)}=\beta \cdot\left|x_{i}(t)-m_{i}^{I}\right|
\end{aligned}
$$

where $\beta$ is an overlap coefficient, which we set to $\beta=0.6$.

\section{Parameter Learning}

The parameter learning phase proceeds after the structure learning phase for each datum. All free RSEFNN parameters are adjusted on-line regardless of whether the corresponding fuzzy rules have been newly generated or previously existed. In parameter learning, all parameters in both the antecedent and consequent components of the fuzzy rules are learned via the on-line gradient descent algorithm using metrics derived from the incoming patterns.

Let us consider the single-output case for simplicity; then, our goal is to minimize the error cost function

$$
E(t+1)=\frac{1}{2}\left(y(t+1)-y^{d}(t+1)\right)^{2}
$$

The consequent parameter vector is updated as follows:

$$
\begin{aligned}
& a_{i j}(t+1)=a_{i j}(t)-\eta \frac{\partial E(t+1)}{\partial a_{i j}(t)} \\
& \frac{\partial E(t+1)}{\partial a_{i j}(t)}=\left(y(t+1)-y^{d}(t+1)\right) \frac{O_{i}^{(3)} u_{j}^{(4)}}{\sum_{k=1}^{r(t)} O_{k}^{(3)}}
\end{aligned}
$$

where $\eta \in(0,1)$ is a learning constant. Each recurrent parameter $\lambda_{q}^{j}$ is updated as follows: 


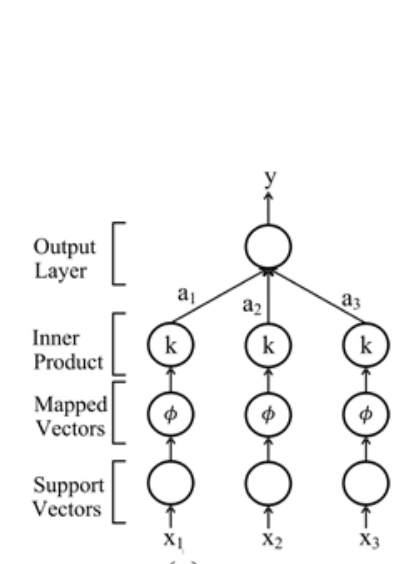

(a)

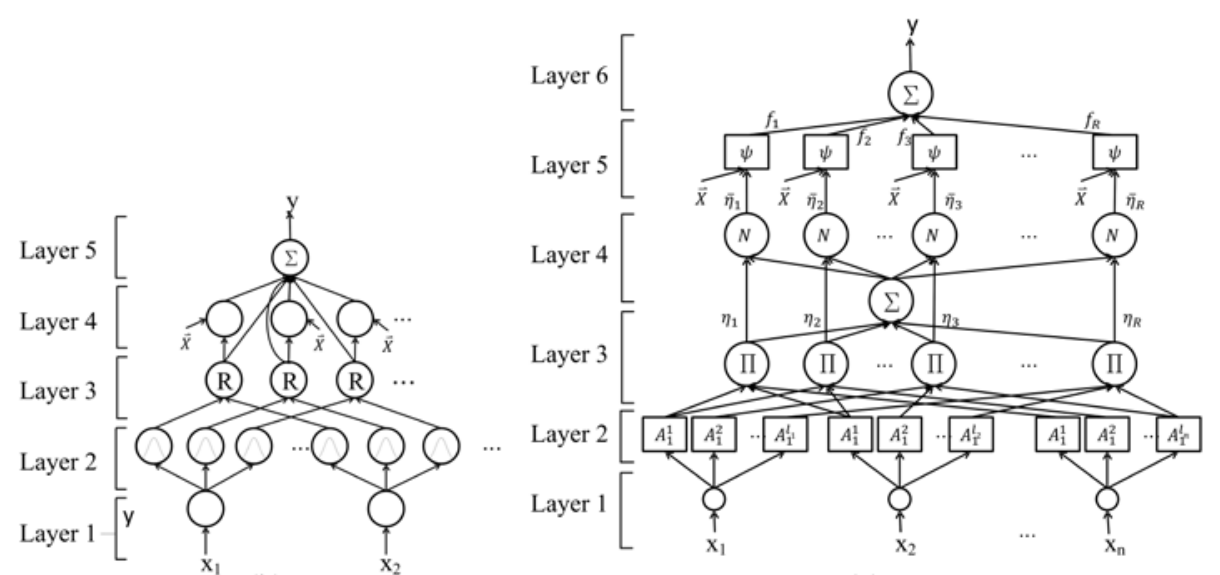

(b)

(c)

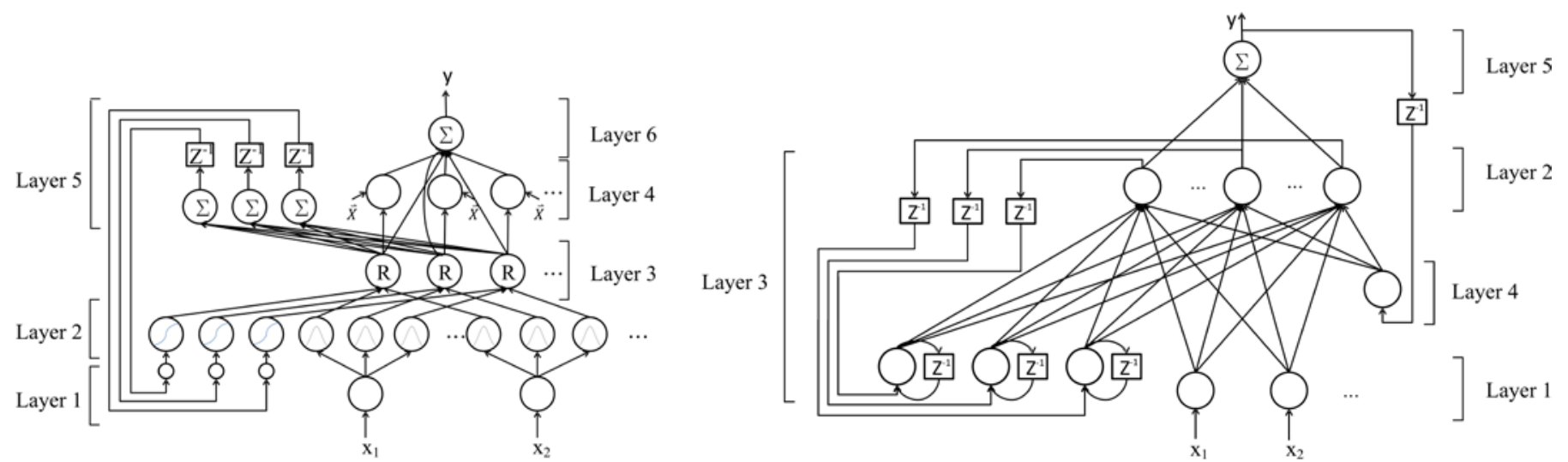

(d)

(e)

Fig. 6. Structures of drowsiness prediction models: (a) support vector regression (SVR), (b) self-organizing neural fuzzy inference network (SONFIN), (c) fuzzy wavelet neural network (FWNN), (d) TSK-type recurrent fuzzy network (TRFN) and (e) recurrent wavelet-based Elman neural network (RWENN).

$$
\begin{aligned}
& \lambda_{q}^{i}(t+1)=\lambda_{q}^{i}(t)-\eta \frac{\partial E}{\partial \lambda_{q}^{i}} \\
& \begin{aligned}
\frac{\partial E}{\partial \lambda_{q}^{i}} & =\frac{\partial E}{\partial y_{q}} \cdot \frac{\partial y_{q}}{\partial \phi_{q}^{i}} \cdot \frac{\partial \phi_{q}^{i}}{\partial \lambda_{q}^{i}} \\
& =\left(y-y^{d}\right) \cdot\left(F^{i}(t)-\phi_{q}^{i}(t-1)\right) \cdot \frac{\omega^{i}-y}{\sum_{i=1}^{r} \phi_{q}^{i}}
\end{aligned}
\end{aligned}
$$

The mean of the Gaussian membership function is updated as follows:

$$
\begin{aligned}
m_{j}^{i}(t+1)=m_{j}^{i}(t)-\eta \frac{\partial E}{\partial m_{j}^{i}} & \begin{aligned}
\frac{\partial E}{\partial m_{q}^{i}} & =\frac{\partial E}{\partial y_{q}} \cdot \frac{\partial y_{q}}{\partial \phi_{q}^{i}} \cdot \frac{\partial \phi_{q}^{i}}{\partial F^{i}} \cdot \frac{\partial F^{i}}{\partial m_{q}^{i}} \\
& =\left(y-y^{d}\right) \cdot \frac{\omega^{i}-y}{\sum_{i=1}^{r} \phi_{q}^{i}} \cdot F^{i} \cdot \lambda_{q}^{i} \cdot \frac{2\left(x_{j}-m_{j}^{i}\right)}{\left(\sigma_{j}^{i}\right)^{2}}
\end{aligned}
\end{aligned}
$$

The variance of the Gaussian membership function is updated as follows:

$$
\begin{aligned}
\sigma_{j}^{i}(t+1)=\sigma_{j}^{i}(t)-\eta \frac{\partial E}{\partial \sigma_{j}^{i}} \\
\begin{aligned}
\frac{\partial E}{\partial \sigma_{j}^{i}} & =\frac{\partial E}{\partial y_{q}} \cdot \frac{\partial y_{q}}{\partial \phi_{q}^{i}} \cdot \frac{\partial \phi_{q}^{i}}{\partial F^{i}} \cdot \frac{\partial F^{i}}{\partial \sigma_{j}^{i}} \\
& =\left(y-y^{d}\right) \cdot \frac{\omega^{i}-y}{\sum_{i=1}^{r} \phi_{q}^{i}} \cdot F^{i} \cdot \lambda_{q}^{i} \cdot \frac{2\left(x_{j}-m_{j}^{i}\right)^{2}}{\left(\sigma_{j}^{i}\right)^{3}}
\end{aligned}
\end{aligned}
$$

\section{Drowsiness Prediction Models}

In this study, the proposed system was compared with the state-of-the-art systems: 1) SVR, 2) SONFIN, 3) FWNN, 4) TRFN and 5) RWENN. The recorded EEG data from the occipital lobe $\left(\mathrm{O}_{1}, \mathrm{O}_{2}\right.$ and $\left.\mathrm{O}_{\mathrm{z}}\right)$ were used to compare the predictive performance of the various systems. The following section briefly describes the structure of each predictor.

\section{A. Support Vector Regression}

Support vector machines (SVMs) constitute a popular machine learning approach to classification, regression and a variety of learning tasks. Support vector regression (SVR) is a means of using an SVM to solve problems regarding function 
TABLE I

LANE-DEPARTURE EVENTS IN A 90-MinUte PERIOD FOR EACH PARTICIPANT

\begin{tabular}{|c|c|c|c|c|c|c|c|c|c|c|c|c|c|c|c|c|c|c|c|c|c|}
\hline Subj. & 1 & 2 & 3 & 4 & 5 & 6 & 7 & 8 & 9 & 10 & 11 & 12 & 13 & 14 & 15 & 16 & 17 & 18 & 19 & 20 & Avg. \\
\hline Trials & 292 & 459 & 413 & 242 & 262 & 291 & 303 & 443 & 180 & 227 & 401 & 337 & 248 & 156 & 286 & 273 & 401 & 179 & 232 & 237 & 293.1 \\
\hline
\end{tabular}

approximation and regression estimation. Fig. 6(a) illustrates the structure of SVR. The primary task of SVR is to search for the hyperplane with the maximum separation property to achieve the highest level of generalization; therefore, the training procedure for SVR is complex, and the computational costs are high. In this study, we adopted the LIBVM library to implement the SVR model and used the radial basis function as its kernel function [31].

\section{B. Self-organizing Neural Fuzzy Inference Network}

A self-organizing neural fuzzy inference network (SONFIN) [32] consists of five layers, as shown in Fig. 6(b), which is a powerful network with both structure and parameter learning abilities. The SONFIN develops from a series of fuzzy IF-THEN rules with Takagi-Sugeno-Kang (TSK)-type consequent parts. This type of network is a well-known feedforward structure, but it cannot naturally address time-varying systems at a high level of performance.

\section{Fuzzy Wavelet Neural Network}

A fuzzy wavelet neural network (FWNN) [33] consists of six layers and combines a TSK-type fuzzy system with wavelet functions, as shown in Fig. 6(c). By virtue of the multiresolution property of the wavelet functions, an FWNN can achieve global and local optimization in both the time and frequency domains in a simple manner [37]. In an FWNN, a gradient algorithm is employed to find the optimal values of the translation and dilation parameters of the wavelet functions, the weights, and the constant terms of the FWNN model.

\section{TSK-type Recurrent Fuzzy Network}

A TSK-type recurrent fuzzy network (TRFN) [30] consists of six layers and utilizes a recurrent structure in the hidden layer to develop a series of recurrent fuzzy rules in consequent components, as illustrated in Fig. 6(d). The recurrent property reflects the information derived from previous firing strengths of IF-THEN rules. In a TRFN, each internal variable is responsible for memorizing the temporal history of its corresponding fuzzy rule; therefore, we use the TRFN model as one of the models for comparison with our proposed approach. A TRFN can also process temporal sequences by means of its recurrent memory network.

\section{E. Recurrent Wavelet-based Elman Neural Network}

A recurrent wavelet-based Elman neural network (RWENN) [34] comprises five layers in which all of the hidden neurons use different wavelet functions as activation functions, thereby significantly increasing the search space. The

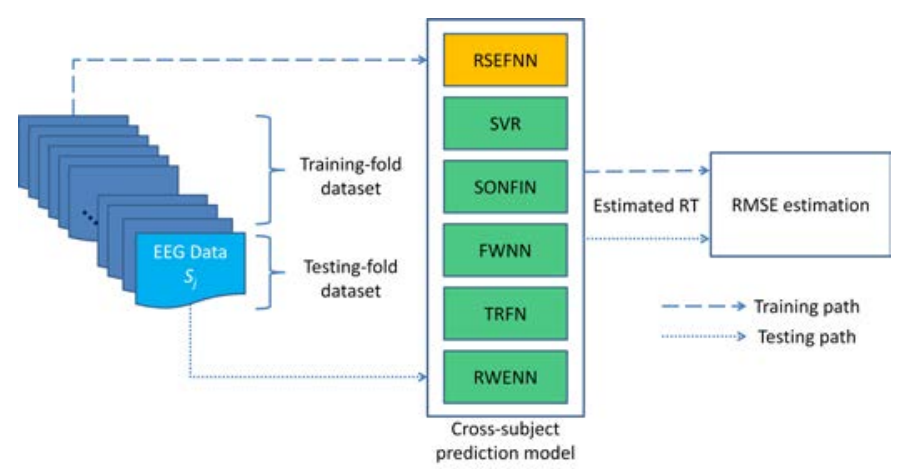

Fig. 7. Generalized cross-subject prediction model for driving fatigue, where Sj represents the $j$-th subject used to generate the test set, whereas the remaining subjects were used for the training set.

structure of the RWENN model is depicted in Fig. 6(e). The feedback from the output layer decreases the time required to reach convergence, and the self-connections of the context neurons enable the memorization of temporal history information. The RWENN approach has been successfully used to learn a nonlinear function in the close-loop control of a permanent-magnet synchronous motor.

\section{EXPERIMENTAL RESULTS AND DISCUSSION}

We collected EEG data from 20 healthy participants, who had been informed and trained prior to the VR-based driving experiment. For each participant, the number of lane-departure events within a period of 90 minutes is listed in Table I, and the behavioral performance of each participant was monitored via a surveillance camera to ensure that fatigue driving was being demonstrated during the driving task. The average number of trials among all 20 participants in the 90-minute driving task was 293.1. Subsequently, the acquired EEG data were preprocessed using EEGLAB for artifact removal, downsampling and segmentation. For the purpose of prediction, we used the brain dynamics recorded $5 \mathrm{~s}$ before each lane-departure event; this information was referred to as the pre-event EEG data, as described in section III-A. Then, the recorded EEG data were transformed into the frequency domain using an FFT to observe the spectral dynamics occurring during the driving task. Based on [11], [14], [16], four spectral bands (delta, theta, alpha and beta) of data from the occipital lobe $\left(\mathrm{O}_{1}, \mathrm{O}_{2}\right.$ and $\left.\mathrm{O}_{\mathrm{z}}\right)$ were used to compare the predictive performance of the various systems (RSEFNN, SVR, SONFIN, FWNN, TRFN and RWENN). To demonstrate practical effectiveness for general users, the proposed system should exhibit both universality and accuracy based on common features as well as applicability to all participants. Thus, the experimental analysis was performed using the cross-subject model to verify that the predictive model was effective in terms of generalizability. 


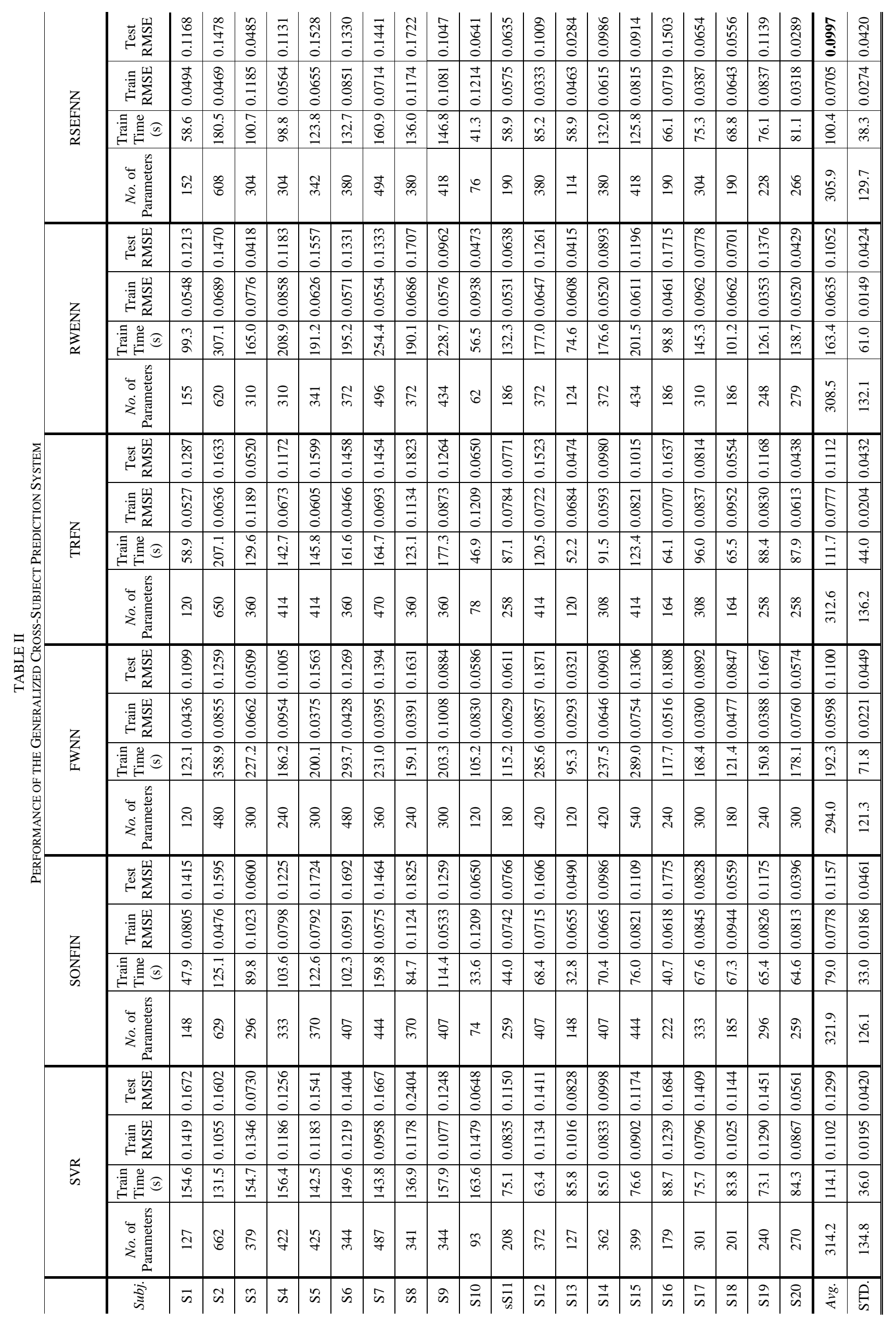




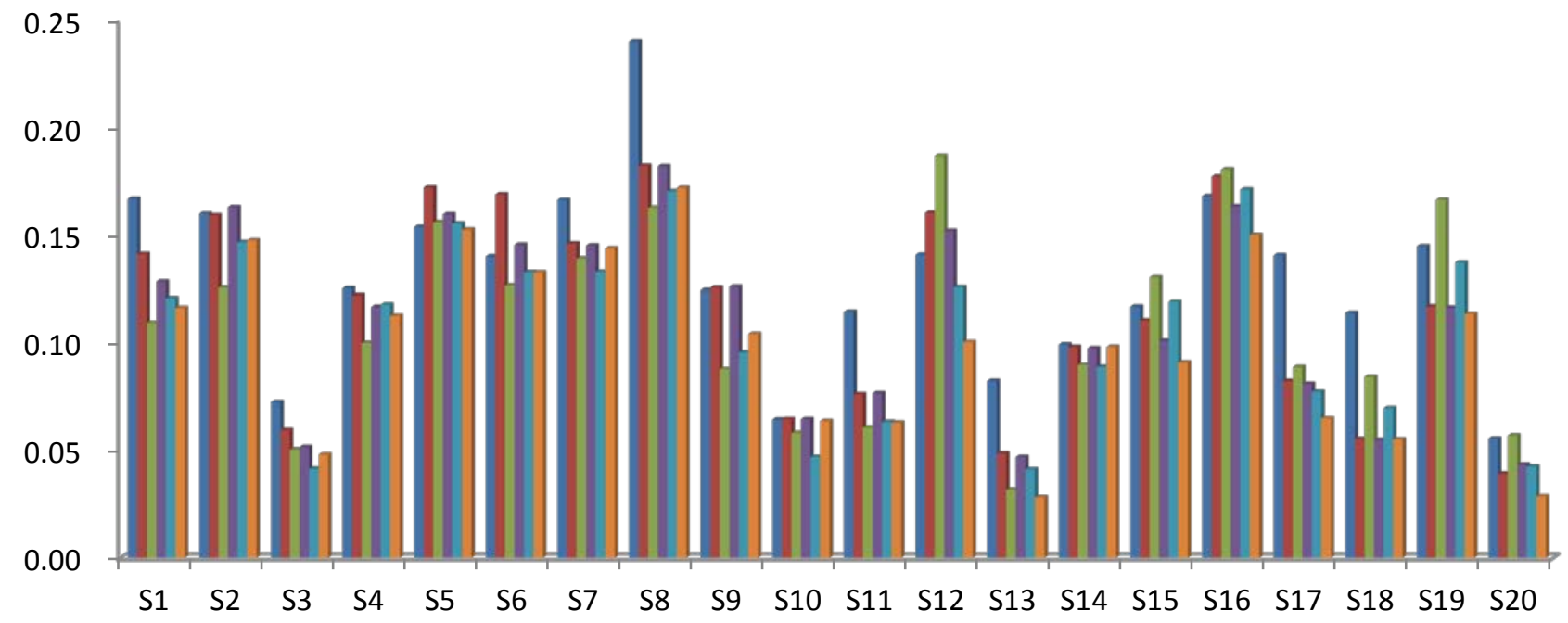

Fig. 8. Test RMSEs for the generalized cross-subject prediction system.

\section{A. Evaluation Results}

The generalized procedure used in the proposed system for cross-subject drowsiness prediction, as summarized in Fig. 7, is discussed in this section. For each model considered in the comparison, the data for one subject were selected as the test set, whereas the data from the remaining subjects were employed to construct the training dataset. Because the EEG data were treated as temporal sequences, the EEG trials in the training set were integrated over different permutations of subjects. For example, given that we collected data from 20 subjects (subject 1 to subject 20) using the EEG device, suppose that we utilized the data from subject 1 , denoted by $\mathrm{S} 1$, as the test set; then, the data from the remaining subjects, who could be rearranged in random orders ([S2 S3 ... S20], [S3 S20 ...S19], etc.) to construct the training dataset, were used to train the system. To evaluate the performance of each model, this process was repeated 10 times, and the average result for each model was compared against the same measure for the other methods.

Table II and Fig. 8 compare the results of the generalized cross-subject prediction system obtained using the considered models (SVR, SONFIN, FWNN, TRFN, RWENN and RSEFNN). The test root-mean-square errors (RMSEs) of the models were $0.1299 \pm 0.0420,0.1157 \pm 0.0461,0.1100 \pm 0.0449$, $0.1112 \pm 0.0432, \quad 0.1052 \pm 0.0424$ and $0.0997 \pm 0.0420$, respectively. Fig. 9 shows boxplots of the test RMSEs for all 20 subjects; the RSEFNN model yielded the lowest RMSEs for the driving task. The estimated RTs for subjects 3, 6 and 8 obtained using the six prediction models are presented in Fig. 10. In Fig. 10 , it is apparent that the RSEFNN model yielded the superior RT estimations in both the high- and low-DS cases. Therefore, the generalized cross-subject prediction system based on the RSEFNN model demonstrates remarkable performance compared with the other models.

\section{B. Discussion}

The results presented in this study indicate that the RSEFNN

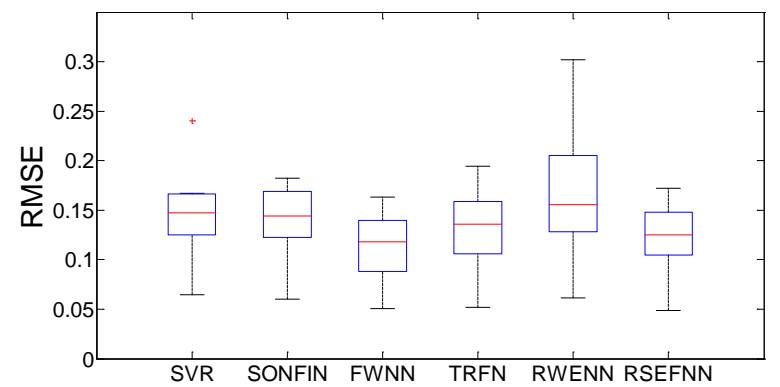

Fig. 9. Boxplots of the test RMSEs for the generalized cross-subject prediction system.

model is both accurate and robust and thus is suitable for use in real-time BCI designs. For all 20 experimental subjects, the RSEFNN was the highest performing model in terms of the average RMSE. Compared with the other considered models, the RSEFNN also incurs lower computational loads in terms of time consumption during the training phase. However, the performance of such a model may differ considerably between subjects; therefore, we verified the proposed system using a cross-subject model. The inter-subject variability that is known to arise in EEG-based BCIs is most likely caused by fluctuations in various processes, such as emotion, memory load and spontaneous cognitive processes, and is likely to be overlooked in group analyses. In previous studies [38], [39], the applicability of BCIs has typically been limited by inter-subject differences, i.e., they were only applied to specific users. In this study, however, our experimental results from the cross-subject model indicate that the RSEFNN model exhibits better performance than the other considered models when applied to all subjects.

To further illustrate this finding, we separated all of the subjects into two groups based on their levels of DS variation: the steady DS group (subjects 3, 10,11, 13, 17, 18 and 20) and the severe DS group (subjects 1-2, 4-9, 12, 14-16 and 19). The subjects in the steady DS group exhibited slower and smoother 

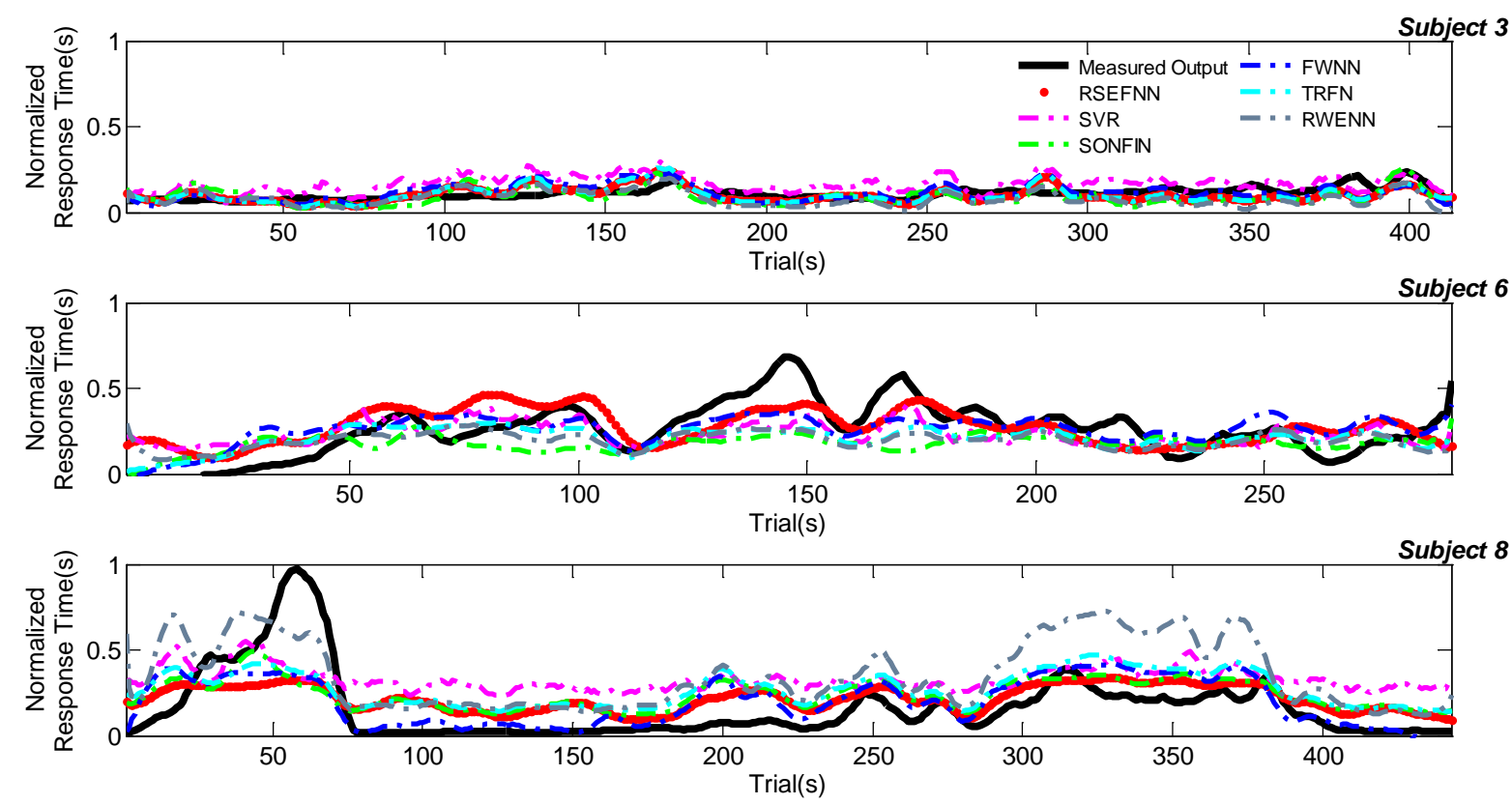

Fig. 10. Evaluation of the prediction curves obtained using the generalized cross-subject models (RSEFNN, SVR, SONFIN, FWNN, TRFN and RWENN) for subjects 3, 6 and 8. The real measured response times, labeled as Measured Output, are presented as black lines. The red dotted lines and the purple, green, blue, cyan and gray dashed lines represent the prediction results of the RSEFNN, SVR, SONFIN, FWNN, TRFN and RWENN models, respectively.

DS variations; the DS fluctuations in this group were similar to those shown in the plots of the data from subject 3 shown in Fig. 10. The cognitive states of the participants in this group remained approximately steady, i.e., they did not suffer fatigue during the driving task. This is the simplest case for all prediction models to address in the recognition task because of the smoothness of the DS variations; therefore, the performances of the various models were all excellent and similar.

By contrast, the subjects in the severe DS group exhibited spontaneous DS variations during the driving task; these DS fluctuations were similar to those shown for subjects 6 and 8 in Fig. 10. The participants suffered from severe fatigue symptoms during the task; therefore, it was difficult for all models to predict their cognitive states. However, the RSEFNN model nevertheless exhibited better performance than the other models because of its local memorization capability.

To demonstrate the improvement achieved by employing the recurrent structure, we also separated the models considered in this study into two categories: current and recurrent models. In the current models, the structure utilizes only the information available from the current inputs and does not possess the ability to retain past information. The SVR, SONFIN and FWNN methods considered in this study are all current models. By contrast, the TRFN, RWENN and RSEFNN models were developed with recurrent components, which exploit the memory capability to gain access to detailed information regarding both current and previous states. The average RMSEs that were obtained using the current models (SVR, SONFIN and FWNN) and recurrent models (TRFN, RWENN and RSEFNN) were 0.1185 and 0.1054 , respectively. This finding suggests that a recurrent model can indeed offer adaptive capabilities based on all available historical information when addressing EEG identification problems.

\section{CONCLUSION}

The brain dynamics of human beings are fundamentally mercurial and varying in time; therefore, the magnitude of the EEG signal recorded during a driving task typically exhibits extreme fluctuations. In previous studies, researchers have generally ignored the fact that an individual's cognitive state is a product of a continuous and cumulative process; instead they have regarded each cognitive event as independent. To improve performance in real applications, the use of a recurrent structure is effective because it endows a neural network with the ability to remember past events. In this paper, we compare the results obtained using both recurrent structures and non-recurrent structures, and it is found that systems with recurrent structures do indeed exhibit significantly improved performance.

To verify the generalizability of the method, a cross-subject approach was utilized to assess the effectiveness of the proposed prediction system based on a recurrent architecture. The performance of an RSEFNN-based system was evaluated using the generalized cross-subject approach, and the results indicated that the RSEFNN model was superior to the SVR, SONFIN, FWNN, TRFN and RWENN models. Based on the presented experimental results, it is clear that our system can effectively process time-varying signals such as EEG data and demonstrates remarkable performance when used for predicting cognitive states of drivers during a car driving task.

\section{REFERENCES}

[1] J. D. Lee, "Fifty years of driving safety research," Hum. Factors, vol. 50, no. 3, pp. 521-528, 2008.

[2] N. Aksan, S. W. Anderson, J. D. Dawson, A. M. Johnson, E. Y. Uc, and M. Rizzo, "Cognitive functioning predicts driver safety on road tests 1 and 2 years later,” J. Am. Geriatr. Soc., vol. 60, no. 1, pp. 99-105, 2012. 
[3] P. H. Ting, J. R. Hwang, J. L. Doong, and M. C. Jeng, "Driver fatigue and highway driving: A simulator study,” Physiol. Behav., vol. 94, no. 3, pp. 448-453, 2008.

[4] E. R. Dahlen, R. C. Martin, K. Ragan, and M. M. Kuhlman, "Driving anger, sensation seeking, impulsiveness, and boredom proneness in the prediction of unsafe driving," Accid. Anal. Prev., vol. 37, no. 2, pp. 341-348, 2005.

[5] I. Karl, G. Berg, F. Ruger, and B. Farber, "Driving Behavior and Simulator Sickness While Driving the Vehicle in the Loop: Validation of Longitudinal Driving Behavior,” IEEE Intell. Transp. Syst. Mag., vol. 5, pp. 42-57, Jan. 2013.

[6] Q. Ji, Z. Zhu, and P. Lan, "Real-time nonintrusive monitoring and prediction of driver fatigue," IEEE Trans. Veh. Technol., vol. 53, pp. 1052-1068, Jul. 2004.

[7] A. Jaimes and N. Sebe, "Multimodal human-computer interaction: A survey,” Comput. Vis. Image Underst., vol. 108, no. 1-2, pp. 116-134, 2007.

[8] A. Nijholt and D. Tan, "Brain-computer interfacing for intelligent systems,” IEEE Intell. Syst., vol. 23, pp. 72-79, May 2008.

[9] Vaughan, Heetderks, Trejo, Rymer, Weinrich, Moore, Kübler, Dobkin, N. Birbaumer, Donchin, and Wolpaw, "Brain-computer interface technology: a review of the Second International Meeting," IEEE Trans. Neural Syst. Rehabil. Eng., vol. 11, pp. 94-109, Jun. 2003.

[10] C. T. Lin, S. F. Tsai, and L. W. Ko, "EEG-Based Learning System for Online Motion Sickness Level Estimation in a Dynamic Vehicle Environment," IEEE Trans. Neural Netw. Learn. Syst., vol. 24, pp. 1689-1700, Oct. 2013.

[11] F. C. Lin, L. W. Ko, C. H. Chuang, T. P. Su, and C. T. Lin, "Generalized EEG-Based Drowsiness Prediction System by Using a Self-Organizing Neural Fuzzy System,” IEEE Trans. Circuits Syst. I Regul. Pap., vol. 59, pp. 2044-2055, Sep. 2012.

[12] J. Long, Y. Li, H. Wang, T. Yu, J. Pan, and F. Li, “A hybrid brain computer interface to control the direction and speed of a simulated or real wheelchair,” IEEE Trans. Neural Syst. Rehabil. Eng., vol. 20, pp. 720-729, Sep. 2012.

[13] M. Azarnoosh, A. Motie Nasrabadi, M. R. Mohammadi, and M. Firoozabadi, "Investigation of mental fatigue through EEG signal processing based on nonlinear analysis: Symbolic dynamics," Chaos Solitons Fractals, vol. 44, no. 12, pp. 1054-1062, 2011.

[14] R. S. Huang, T. P. Jung, A. Delorme, and S. Makeig, "Tonic and phasic electroencephalographic dynamics during continuous compensatory tracking," Neuroimage, vol. 39, no. 4, pp. 1896-1909, 2008.

[15] S. K. L. Lal and A. Craig, "A critical review of the psychophysiology of driver fatigue,” Biol. Psychol., vol. 55, no. 3, pp. 173-194, 2001.

[16] C. H. Chuang, L. W. Ko, T. P. Jung, and C. T. Lin, "Kinesthesia in a sustained-attention driving task," Neuroimage, vol. 91, no. 1, pp. 187-202, 2014.

[17] F. Lotte, M. Congedo, A. Lécuyer, F. Lamarche, and B. Arnaldi, “A review of classification algorithms for EEG-based brain-computer interfaces," J. Neural Eng., vol. 4, no. 2, pp. 1-13, 2007.

[18] N. Acir, I. Oztura, M. Kuntalp, B. Baklan, and C. Guzelis, “Automatic detection of epileptiform events in EEG by a three-stage procedure based on artificial neural networks," IEEE Trans. Biomed. Eng., vol. 52, pp. 30-40, Jan. 2005.

[19] U. Orhan, M. Hekim, and M. Ozer, "EEG signals classification using the K-means clustering and a multilayer perceptron neural network model," Expert Syst. Appl., vol. 38, no. 10, pp. 13475-13481, 2011.

[20] S. Ghosh Dastidar, H. Adeli, and N. Dadmehr, "Principal component analysis-enhanced cosine radial basis function neural network for robust epilepsy and seizure detection," IEEE Trans. Biomed. Eng., vol. 55, pp. 512-518, Feb. 2008.

[21] W. Y. Hsu, "Fuzzy Hopfield neural network clustering for single-trial motor imagery EEG classification,” Expert Syst. Appl., vol. 39, no. 1, pp. 1055-1061, 2012.

[22] D. Coyle, G. Prasad, and T. M. McGinnity, "Faster Self-Organizing Fuzzy Neural Network Training and a Hyperparameter Analysis for a Brain-Computer Interface," IEEE Trans. Syst. Man Cybern. Part B-Cybern., vol. 39, pp. 1458-1471, Dec. 2009.

[23] A. Erfanian and B. Mahmoudi, "Real-time ocular artifact suppression using recurrent neural network for electro-encephalogram based brain-computer interface," Med. Biol. Eng. Comput., vol. 43, no. 2, pp. 296-305, 2005.

[24] E. D. Übeyli, "Analysis of EEG signals by implementing eigenvector methods/recurrent neural networks,” Digit. Signal Process., vol. 19, no. 1, pp. 134-143, 2009.
[25] N. Guler, E. Ubeyli, and I. Guler, "Recurrent neural networks employing Lyapunov exponents for EEG signals classification," Expert Syst. Appl., vol. 29, no. 3, pp. 506-514, 2005.

[26] P. R. Davidson, R. D. Jones, and M. T. Peiris, "Detecting behavioral microsleeps using EEG and LSTM recurrent neural networks," in Proceedings of the 27th Annual International Conference of the Engineering in Medicine and Biology Society, Shanghai, China, Jan. 2006, pp. 5754-5757.

[27] C. T. Lin, L. W. Ko, I. F. Chung, T. Y. Huang, Y. C. Chen, T. P. Jung, and S. F. Liang, "Adaptive EEG-based alertness estimation system by using ICA-based fuzzy neural networks," IEEE Trans. Circuits Syst. I Regul. Pap., vol. 53, pp. 2469-2476, Nov. 2006.

[28] C. F. Juang, Y. Y. Lin, and C. C. Tu, “A recurrent self-evolving fuzzy neural network with local feedbacks and its application to dynamic system processing,” Fuzzy Sets Syst., vol. 161, no. 19, pp. 2552-2568, 2010.

[29] C. J. Lin and C. C. Chin, "Prediciton and identification using wavelet-based recurrent fuzzy neural networks," IEEE Trans. Syst. Man Cybern. Part B-Cybern., vol. 34, pp. 2144-2154, Oct. 2004.

[30] C. F. Juang, “A TSK-type recurrent fuzzy network for dynamic systems processing by neural network and genetic algorithms," IEEE Trans. Fuzzy Syst., vol. 10, no. 2, pp. 155-170, Apr. 2002.

[31] C. C. Chang and C. J. Lin, "LIBSVM: a library for support vector machines," ACM Trans. Intell. Syst. Technol., vol. 2, no. 3, pp. 1-39, 2011.

[32] C. F. Juang and C. T. Lin, "An on-line self-constructing neural fuzzy inference network and its applications," IEEE Trans. Fuzzy Syst., vol. 6, pp. 12-32, Feb. 1998.

[33] S. Yilmaz and Y. Oysal, "Fuzzy wavelet neural network models for prediction and identification of dynamical systems.," IEEE Trans. Neural Netw., vol. 21, pp. 1599-1609, Oct. 2010.

[34] F. F. M. El-Sousy, "Intelligent Optimal Recurrent Wavelet Elman Neural Network Control System for Permanent-Magnet Synchronous Motor Servo Drive," IEEE Trans. Ind. Informatics, vol. 9, pp. 1986-2003, Nov. 2013.

[35] A. Delorme and S. Makeig, "EEGLAB: An open source toolbox for analysis of single-trial EEG dynamics including independent component analysis," J. Neurosci. Methods, vol. 134, no. 1, pp. 9-21, 2004.

[36] Z. Li, M. Hayashibe, C. Fattal, and D. Guiraud, "Muscle Fatigue Tracking with Evoked EMG via Recurrent Neural Network: Toward Personalized Neuroprosthetics,” IEEE Comput. Intell. Mag., vol. 9, pp. 38-46, May 2014.

[37] D. W. C. Ho, P. A. Zhang, and J. Xu, "Fuzzy wavelet networks for function learning,” IEEE Trans. Fuzzy Syst., vol. 9, pp. 200-211, Feb. 2001.

[38] B. Blankertz, G. Dornhege, M. Krauledat, K. R. Müller, V. Kunzmann, F. Losch, and G. Curio, "The Berlin brain-computer interface: EEG-based communication without subject training," IEEE Trans. Neural Syst. Rehabil. Eng., vol. 14, pp. 147-152, Jun. 2006.

[39] J. Hlinka, C. Alexakis, A. Diukova, P. F. Liddle, and D. P. Auer, "Slow EEG pattern predicts reduced intrinsic functional connectivity in the default mode network: An inter-subject analysis," Neuroimage, vol. 53, no. 1, pp. 239-246, 2010.

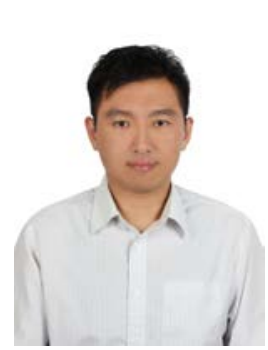

Yu-Ting Liu received the B.S. degree in Department of Electrical and Control Engineering from National Chiao-Tung University, Hsinchu, Taiwan, in 2010. He is currently pursuing the Ph.D. degree with the Institute of Electrical and Control Engineering, National Chiao Tung University, Hsinchu, Taiwan.

His current research interests include fuzzy logic theory, machine learning, computer vision, EEG analysis and application. 


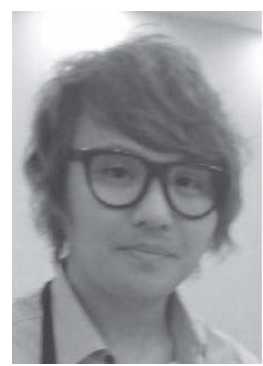

Yang-Yin Lin (M'13) received the M.S. degree from the Institute of Electrical Engineering, National Chung Hsing University, Taichung, Taiwan, in 2008, and the Ph.D. degree from the Department of Electrical Engineering, National Chiao Tung University (NCTU), Hsinchu, Taiwan, in 2013.

$\mathrm{He}$ is currently with the Electronic Systems Research Division, National Chung-Shan Institute of Science and Technology (NCSIST), as an Assistant Researcher. His current research interests include evolutionary computation, type- 2 neural fuzzy systems, deep learning, transfer fuzzy learning, and Field-programmable gate array (FPGA) chip design based on neural network architecture.
Conference on Fuzzy Systems. Dr. Lin is the coauthor of Neural Fuzzy Systems (Prentice-Hall), and the author of Neural Fuzzy Control Systems with Structure and Parameter Learning (World Scientific). He has published more than 200 journal papers (Total Citation: 18,438, H-index: 51, i10-index: 301) in the areas of neural networks, fuzzy systems, multimedia hardware/software, and cognitive neuro-engineering, including approximately 97 IEEE journal papers.

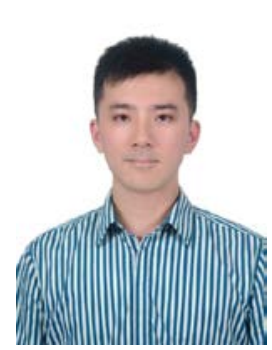

Shang-Lin Wu was born in Kaohsiung, Taiwan, in 1987. He received the B.S. and M.S. degree in Department of Computer Science and Information Engineering from National Chiayi University, Chiayi, Taiwan, R.O.C., in 2009, and 2011, respectively. He is currently working toward the Ph.D. degree in Institute of Electrical Control Engineering at National Chiao Tung University (NCTU), Hsinchu, Taiwan, R.O.C.

His research interests are in the areas of fuzzy theory, transfer learning, biomedical signal processing, biofeedback control, and embedded system.

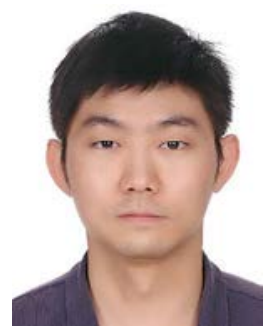

Chun-Hsiang Chuang received the B.S. degree in mathematics education from Taipei Municipal Teachers College in 2004, the M.S. degree in educational measurement and statistics from National Taichung University in 2009, and the Ph.D. degree in Electrical Engineering from National Chiao Tung University (NCTU) in 2014. He was a visiting scholar at Swartz Center for Computational Neuroscience, University of California in 2012-2013. Currently, he is a postdoctoral researcher of Brain Research Center at NCTU, Taiwan.

His research interests include machine learning, computational neuroscience, biomedical signal processing, and brain-computer interface.

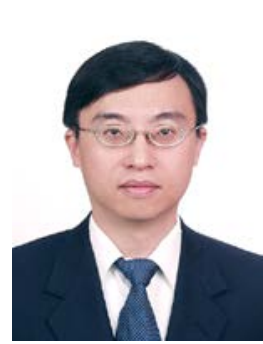

Chin-Teng Lin (S'88-M'91-SM'99-F'05) received the B.S. degree from National Chiao-Tung University (NCTU), Taiwan in 1986, and the Master and Ph.D. degree in electrical engineering from Purdue University, USA in 1989 and 1992, respectively. He is currently the Chair Professor of Electrical and Computer Engineering, Director of Brain Research Center, National Chiao Tung University, International Faculty of University of California at San-Diego (UCSD), Adjunct Professor of University of Technology Sydney, and Honorary Professorship of University of Nottingham. Dr. Lin was elevated to be an IEEE Fellow for his contributions to biologically inspired information systems in 2005, and was elevated International Fuzzy Systems Association (IFSA) Fellow in 2012. He is elected as the Editor-in-chief of IEEE Transactions on Fuzzy Systems since 2011. He also served on the Board of Governors at IEEE Circuits and Systems (CAS) Society in 2005-2008, IEEE Systems, Man, Cybernetics (SMC) Society in 2003-2005, IEEE Computational Intelligence Society in 2008-2010, and Chair of IEEE Taipei Section in 2009-2010. Dr. Lin was the Distinguished Lecturer of IEEE CAS Society from 2003 to 2005. He served as the Deputy Editor-in-Chief of IEEE Transactions on Circuits and Systems-II in 2006-2008. Dr. Lin was the Program Chair of IEEE International Conference on Systems, Man, and Cybernetics in 2005 and General Chair of 2011 IEEE International 